\title{
Reviewers 2013
}

The Zeitschrift für Psychologie expresses its thanks to the following colleagues who contributed to the peer review of manuscripts this year. They reviewed the incoming manuscripts very conscientiously and provided constructive suggestions to many of our authors. Furthermore, in most cases they reviewed quickly, which is a fundamental prerequisite for a rapid manuscript management. The Zeitschrift für Psychologie and its editors are greatly indebted to all of them.

Erik M. Altmann

John W. Berry

Dina Birman

Mark Blades

Fran C. Blumberg

Dankmar Böhning

David Bourguignon

Marcel Brass

Philipp Doebler

Thomas Ehring

Günther Gediga

Hector Grad

James Grange

Ulrike Graßhoff

Eva Green

Sibylle Hailbrunn

Heinz Holling
Gabriel Horenczyk

Jan De Houwer

Ronald Hübner

Inga Jasinskaja-Lahti

Yoav Kessler

Andrea Kiesel

Iring Koch

Axel Kohler

Ankica Kosic

Coen Kouwenhoven

Chan-Hoong Leong

Karmela Liebkind

Baptist Liefooghe

Vanja Ljujic

Jan-Erik Lönnqvist

Nachshon Meiran

Mirjam Moerbeek
Frosso Motti-Stefanidi

Wim Notebaert

Caroline Oates

Sabine Otten

Ron Pat-El

David L. Sam

Vassilis Saroglou

Ricarda Schubotz

Stefanie Schuch

Rainer Schwabe

Peter Titzmann

Aune Valk

Fons van der Vijver

Paul Vedder

Violaine Veen

Kim-Phuong L. Vu 